\title{
What External Variables Affect Sensorimotor Rhythm Brain-Computer Interface (SMR-BCI) Performance?
}

Alex J. Horowitz, DO, ${ }^{1,6}$ Christoph Guger, Dr.techn., ${ }^{2}$ Milena Korostenskaja, PhD ${ }^{1,3,4,5}$

\section{Abstract}

\section{Description}

Sensorimotor rhythm-based brain-computer interfaces (SMR-BCls) are used for the acquisition and translation of motor imagery-related brain signals into machine control commands, bypassing the usual central nervous system output. The selection of optimal external variable configuration can maximize SMR-BCl performance in both healthy and disabled people. This performance is especially important now when the $\mathrm{BCl}$ is targeted for everyday use in the environment beyond strictly regulated laboratory settings. In this review article, we summarize and critically evaluate the current body of knowledge pertaining to the effect of the external variables on SMR-BCl performance. When assessing the relationship between SMR-BCl performance and external variables, we broadly characterize them as elements that are less dependent on the $\mathrm{BCl}$ user and originate from beyond the user. These elements include such factors as $\mathrm{BCl}$ type, distractors, training, visual and auditory feedback, virtual reality and magneto electric feedback, proprioceptive and haptic feedback, carefulness of electroencephalography (EEG) system assembling and positioning of EEG electrodes as well as recording-related artifacts. At the end of this review paper, future developments are proposed regarding the research into the effects of external variables on $\mathrm{SMR}-\mathrm{BCl}$ performance. We believe that our critical review will be of value for academic $\mathrm{BCl}$ scientists and developers and clinical professionals working in the field of $\mathrm{BCls}$ as well as for SMR-BCl users.

\section{Keywords}

adoption rates; auditory feedback; brain-computer interfaces ( $\mathrm{BCls}$ ); $\mathrm{BCl}$ accuracy; $\mathrm{BCl}$ literacy; $\mathrm{BCl}$ optimization; $\mathrm{BCl}$ performance; classification accuracy; distractors; dry electrodes; drone control; electrocorticography (ECoG); electroencephalography (EEG); EEG artifacts; EEG waveforms; external factors; external variables; event-related potentials; exoskeleton; haptic feedback; information transfer rate (ITR); internal factors; internal variables; magnetoencephalography (MEG); motor imagery; neurophysiology; odd-ball paradigm; P300 response; sensorimotor rhythm (SMR); sensory feedback; steadystate somatosensory evoked potential (SSSEP); steady-state visually evoked potentials (SSVEPs); motor imagery training; neuroprosthetics; transcranial magnetic stimulation (TMS); repetitive transcranial magnetic stimulation (rTMS); multimodal feedback; virtual reality (VR); vibrotactile feedback; visual feedback

\section{Introduction}

A brain-computer interface $(\mathrm{BCl})$ is a device that records and translates the user's brain activity into various command signals, thus bypassing muscle activity and allowing direct communication between the brain and various devices. Brain activity for $\mathrm{BCl}$ control can be recorded with high millisecond scale temporal resolution through magnetoencephalography
(MEG), electroencephalography (EEG) and electrocorticography (ECoG). ' We limit the scope of this review article to the BCls driven by electrical signals recorded with EEG. The reason behind this choice is that EEG-driven $\mathrm{BCls}$ are the number one target for $\mathrm{BCl}$ translation from the laboratory to real-world settings due to the high temporal resolution of EEG methodology. After capturing the EEG

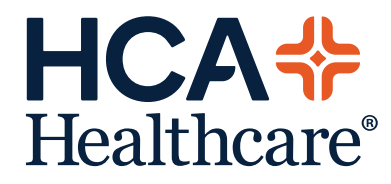

www.hcahealthcarejournal.com

(C) 2021 HCA Physician Services, Inc. d/b/a Emerald Medical Education
HCA Healthcare Journal of Medicine 


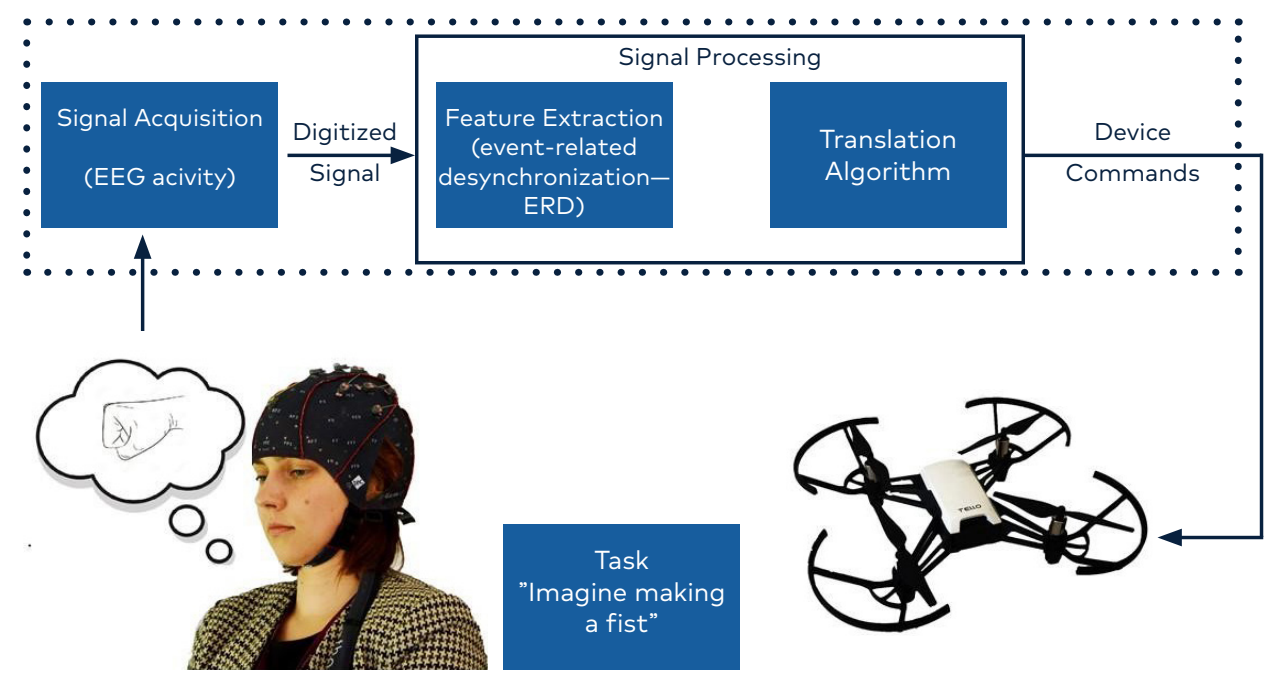

Figure 1. Brain Computer Interface set-up. An electrode array detects $\mathrm{BCl}$ user's electrical brain activity during the motor imagery (for example, imagining making a fist). The $\mathrm{BCl}$ translates the acquired signal according to a fixed or adaptive algorithm, extracting relevant features, for example, event-related desynchronization (ERD). BCl output manifests as the command of a device, such as steering a drone in its flight. (Photographs courtesy of the authors.)

interest, the $\mathrm{BCl}$ processes it by using a pre-defined fixed or changing ("adaptive") algorithm and translates the analyzed signal (its specific features) in real-time into computer commands. (Figure 1)

Among several types of electrical brain signals that can be detected and utilized for $\mathrm{BCl}$ control, sensorimotor rhythm (SMR) is one of the most common. Sensorimotor rhythmbased BCls (SMR-BCls) (also referred to as motor imagery $\mathrm{BCls}$ or $\mathrm{Ml}-\mathrm{BCls}$ ) can detect the event-related desynchronizations (ERD) in electrical activity recorded with an EEG from the sensorimotor brain areas during a motor imagery (MI) task. SMR-BCls hold great potential to advance the field of motor rehabilitation (for review, see Bamdad et al. ${ }^{2}$ ). A systematic review of cohort SMR-BCl studies by Monge-Pereira et al. demonstrated level II evidence that EEG-based SMR-BCl intervention can be a promising rehabilitation approach for upper motor function rehabilitation after stroke. ${ }^{3}$ Moreover, a $\mathrm{BCl}$ may be used as a substitute to overcome functional deficits in individuals with compromised skeletal or motor system functions (such as paralysis and amputation). ${ }^{4}$

With continued development, a future becomes possible where $\mathrm{BCl}$ is a commonplace technology fully incorporated into everyday life in both the clinical population and healthy peo- ple. To achieve this widespread $\mathrm{BCl}$ adoption, it is imperative to understand how the user's internal and external environment impact SMR-BCl performance. Indeed, the performance of an SMR-BCl is largely determined by the efficacy of the user, the $\mathrm{BCl}$ itself and the operational conditions. This review article will focus on the effect of external factors on $\mathrm{BCl}$ performance. External variables are identified as those elements of the environment that mainly reside beyond the SMR-BCl user and within the SMR-BCl itself. Internal variables are defined as those factors largely originating from within the SMR-BCl user. It should be noted that these working definitions of internal and external variables are simply operational and are used for this specific paper only. Variations on these terms can be found elsewhere. In some circumstances, internal and external variables an be used interchangeably. For example, distractibility (originating within the user) and distractors (originating outside the user). Figure 2 provides a flow chart depicting the relationship between internal and external factors with SMR-BCl performance.

Several studies have been conducted in an attempt to isolate some external variables, which may affect any metric of SMR-BCl performance, such as signal information transfer rate (ITR), correct response rate (CRR), adoption rate, classification accuracy and reaching target accuracy (for more details, see Table 1)..$^{5-11}$ 


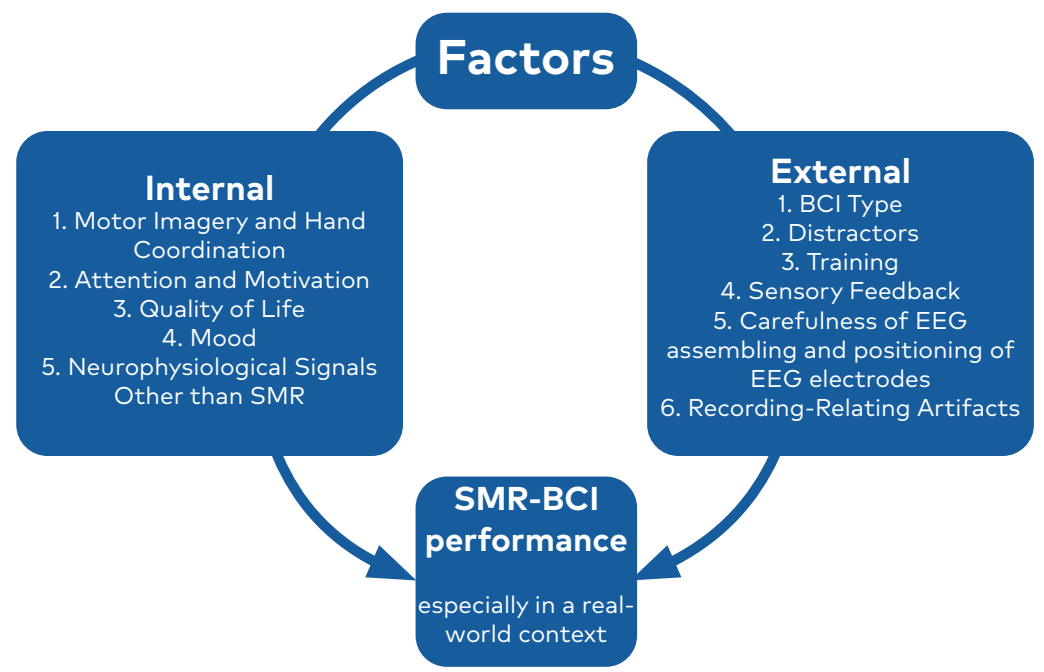

Figure 2. Factors may be divided into external or internal variables. Either may influence SMR-BCI performance. These variables may positively, negatively, or not at all influence users' SMR-BCl performance. ${ }^{76} \mathrm{An}$ SMR-BCl that emphasizes design elements that positively influence SMR-BCI performance. Conversely, an SMR-BCl that also mitigates design elements that negatively influence performance offers great hope for widespread, everyday SMR-BCl use.

The goals of our current review paper are (1) to summarize existing knowledge about the external factors affecting SMR-BCl performance and critically examine the studies on this subject published to date, as well as (2) to discuss limitations and propose further directions of $\mathrm{MI}-\mathrm{BCl}$ research with other possible factors that may or may not affect the SMR-BCl's performance when presented within a real-world context. We think our paper will make a significant contribution to the transition of SMR$\mathrm{BCls}$ from academic laboratories to clinical settings and also have real-world applications. This paper will be of valuable use for clinical $\mathrm{BCl}$ users, as well as academic scientists, clinicians and engineers working with $\mathrm{BCls}$.

\section{External Variables}

External variables in the context of SMR-BCls discussed in the current articles are those that largely originate from within the $\mathrm{BCl}$ system itself or beyond the $\mathrm{BCl}$ user. Examples include $\mathrm{BCl}$ type, distractors, training, sensory feedback, carefulness of EEG assembling and positioning of EEG electrodes, and recording-related artifacts. $\mathrm{BCl}$ types vary in the way they detect and analyze (for example, building a model and performing pattern recognition) specific brain signals. For this reason, the $\mathrm{BCl}$ type is based on the subject's intrinsic brain activity and depends on it. Nevertheless, the choice of a pattern of interest is a decision made by the experimenter rather than $\mathrm{BCl}$ user. Such a decision depends on the purpose behind the $\mathrm{BCl}$ use. It is important to note that the way brain activity is captured within each specific $\mathrm{BCl}$ type can affect $\mathrm{BCl}$ performance. Therefore, we have chosen to categorize the $\mathrm{BCl}$ type as an external variable that influences $\mathrm{BCl}$ performance. Likewise, our selection of distractors as external variables follows a similar interrelated relationship with distractibility. Although distractors are an external variable, they are closely related to distractibility-an internal variable. This introduction is followed by the summaries of studies that have been performed to investigate the effects of external variables on SMR-BCl performance. At the end of our current review, these external variables and their effect on SMR-BCl performance are recapped in Table 1.

\subsection{BCl Type}

$\mathrm{BCl}$ performance relies on specific electrical brain signals of the $\mathrm{BCl}$ user. The detection and analysis of specific EEG waveforms serve as the basis for the different types of BCls. Due to the varying characteristics of these EEG waveforms, it would be expected that different $\mathrm{BCl}$ types would demonstrate varying levels of $\mathrm{BCl}$ performance. A series of studies have been performed by Guger et al. to investigate the adoption rates of P300, steady-state visual evoked potential (SSVEP) and SMR-BCls. ${ }^{8}$ 
Table 1. Summary of External Variables Affecting BCI Performance.

\begin{tabular}{|c|c|c|c|}
\hline $\begin{array}{l}\text { External } \\
\text { Variables }\end{array}$ & Referenced Studies & $\begin{array}{l}\text { Effect on } \mathrm{BCl} \\
\text { Performance }\end{array}$ & Details \\
\hline 1.1 BCI Type & $\begin{array}{l}\text { Brunner et al. (2011);12 } \\
\text { Ding et al. (2006); } ;^{13} \\
\text { Guger et al. (2012); } \\
\text { Guger et al. (2009); } \\
\text { Guger et al. (2003);: } \\
\text { Guger et al. (2015);14 } \\
\text { Guger et al. (2000); } ;^{15} \\
\text { Kapeller et al. (2013); }{ }^{16} \\
\text { Kus et al. (2013);17 } \\
\text { Malone et al. (2014);18 } \\
\text { Musiek et al. (1992);19 } \\
\text { Srinivasan et al. } \\
\text { (2006);20 } \\
\text { Yao et al. (2018);21 } \\
\text { Zhu et al. (2010) }{ }^{22}\end{array}$ & $\begin{array}{l}\text { SSVEP- } \\
\text { BCls have } \\
\text { the highest } \\
\text { adoption } \\
\text { rate }\end{array}$ & $\begin{array}{l}\text { SSVEP-based and P300-based BCls have sim- } \\
\text { ilarly high adoption rates, with SSVEP slightly } \\
\text { higher than P300. SMR-BCIs have low adoption } \\
\text { rates. Adoption rates are influenced by mediat- } \\
\text { ing factors. }\end{array}$ \\
\hline 1.2 Distractors & $\begin{array}{l}\text { Brandl et al. (2016); }{ }^{23} \\
\text { Calabrese (2008); } \\
\text { Chaby et al. (2015);25 } \\
\text { Emami and Chau } \\
\text { (2018); } \\
\text { Friedrich et al. (2011) }\end{array}$ & $\begin{array}{l}\text { Positive } \\
\text { effect }\end{array}$ & $\begin{array}{l}\text { Passive auditory distraction optimized men- } \\
\text { tal imagery-based } \mathrm{BCl} \text { classification accuracy. } \\
\text { Passive auditory distraction was also associated } \\
\text { with the highest P300 amplitudes and shortest } \\
\text { P300 latencies. } \\
\text { Infrequent, small visual distractors altered mu } \\
\text { and beta power of motor imagery-specific } \\
\text { patterns but did not significantly alter SMR-BCl } \\
\text { classification accuracy. }\end{array}$ \\
\hline 1.3 Training & $\begin{array}{l}\text { Kaiser et al. (2014); }{ }^{28} \\
\text { Meng and } \mathrm{He}(2019) ; 29 \\
\text { Pichiorri et al. (2011);30 } \\
\text { Toppi et al. (2014). }{ }^{31}\end{array}$ & $\begin{array}{l}\text { Positive } \\
\text { effect }\end{array}$ & $\begin{array}{l}\text { Results revealed a significant increase in the } \\
\text { group average SMR-BCl classification accuracy } \\
\text { and information transfer rate. } \\
\text { Unique specific spectral and spatial cortical ac- } \\
\text { tivity patterns in response to a motor imagery } \\
\text { training task }\end{array}$ \\
\hline $\begin{array}{l}1.4 .1 \text { Visual } \\
\text { and Auditory } \\
\text { Feedback }\end{array}$ & $\begin{array}{l}\text { Angulo-Sherman and } \\
\text { Gutierrez (2015);32 } \\
\text { Brumberg et al. } \\
\text { (2018);3 } \\
\text { Chaby et al. (2015);25 } \\
\text { McCreadie et al. (2012; } \\
\text { 2014);34,35 } \\
\text { Miller et al. (2010);36 } \\
\text { Ono et al. (2013);37 } \\
\text { Orand et al. (2012);38 } \\
\text { Pichiorri et al. (2011);30 } \\
\text { Sollfrank et al. (2016); } ;^{11} \\
\text { Zich et al. (2015) }\end{array}$ & $\begin{array}{l}\text { Positive } \\
\text { effect }\end{array}$ & $\begin{array}{l}\text { A significant improvement in SMR-BCl classifi- } \\
\text { cation accuracy was associated with the funnel } \\
\text { feedback paradigms relative to the CB para- } \\
\text { digm. } \\
\text { Significant improvement of motor imagery } \\
\text { learning in SMR-BCl users who received ab- } \\
\text { stract visual feedback. } \\
\text { Significantly enhanced motor imagery task-spe- } \\
\text { cific brain activity during feedback conditions } \\
\text { relative to no EEG monitoring feedback. } \\
\text { SMR-BCI users with auditory feedback demon- } \\
\text { strated consistent and sustained enhancements } \\
\text { of average classification accuracy and average } \\
\text { peak classification accuracy. } \\
\text { Optimal SMR-BCl performance may be } \\
\text { achieved when multimodal feedback is consis- } \\
\text { tent with SMR-BCl task goals. }\end{array}$ \\
\hline
\end{tabular}


Table 1. Summary of External Variables Affecting BCI Performance. Cont'd.

External
Variables

.4.2 Virtual

Reality and

Magnetoelec-

tric Feedback

\section{Referenced Studies}

Burin et al. (2019);40

Cho et al. (2016); 41

de Vries et al. (2009);42

Guger et al. (2015);14

Huang et al. (2019);43

Johnson et al. (2018); ${ }^{44}$

Long et al. (2018);45

Pan et al. (2019); ${ }^{46}$

Shu et al. (2018a); ${ }^{47}$

Topper et al. (1999); ${ }^{48}$

Vourvopoulos et al.

(2019); ${ }^{49}$

Yi et al. $(2017)^{50}$

1.4.3 Proprioceptive and Haptic Feedback
Darvishi et al. (2017); ${ }^{51}$

Missiroli et al. (2019); 52

Nakayashiki et al.

(2014); ;3

Penaloza et al. (2018); $;^{54}$

Ramos-Murguialday et al. (2012);10

Shu et al. (2018b); 55

Vukelic and Gharabaghi

(2015); 56

Wang et al. (2019) 57

\section{Effect on BCl} Performance

\section{Positive}

effect

Positive

effect

\title{
Details
}

Increase in the intensity of Ml-related brain activity.

Significant improvement of SMR-BCl performance in stroke patients with high-frequency repetitive transcranial magnetic stimulation sessions.

\begin{tabular}{ll}
\hline $\begin{array}{l}\text { 1.5 Carefulness } \\
\text { of EEG Assem- }\end{array}$ & Baek et al. (2019); $;^{58}$ \\
bling and Posi- & (2015); $;^{59}$ \\
tioning of EEG & Korostenskaja et al. \\
electrodes & (2017); \\
& Lin et al. (2019);60 \\
& Marini et al. (2019); ${ }^{61}$ \\
& Raduntz and Meffert \\
& (2019);62 \\
& Sannelli et al. (2010);63 \\
& Spuler (2017);64 \\
& Zhang et al. (2019)
\end{tabular}

Positive effect

\begin{abstract}
Proprioceptive feedback facilitated motor imagery-related operant learning of SMR beta-band modulation.
\end{abstract}

Motor imagery training significantly improved the percent of time the robotic arm moved, number of robotic arm onsets, and the reaching target accuracy of a neuroprosthesis controlled by an SMR-BCl.

No significant change in SMR-BCl proficiency with vibration at the fingertips relative to controls who received haptic stimulation at the wrist.

Dry-electrode performed comparably to the wet-electrode system.

Trend towards a consistent distance between hand motor area and site of mu-rhythm modulation for optimal EEG-recording electrode, placement. Distance most prevalent mediolaterally.

The performance of a portable EEG smart cap with novel dry active electrodes and novel spatial filtering circuit was validated.

Design of portable SMR-BCI with dry electrodes and a three-dimensional novel convolutional neural network was validated.

\subsection{Record- ing-Related Artifacts}

Frolich et al. (2015); ${ }^{27}$ Nijboer et al. (2010); 9 Winkler et al. (2011); ${ }^{66}$ Yuan and $\mathrm{He}(2014)^{67}$
Negative effect
The ease of over the scalp EEG recording renders this technology more susceptible to artifacts.

Automatic classification algorithm to identify and remove most artifactual independent component analysis source components optimized $\mathrm{SMR}-\mathrm{BCl}$ performance.

Only muscle artifacts negatively influenced the SMR-BCl error rate. This association was eliminated with a centrally arranged electrode array. 
Adoption rate is defined as the proportion of the tested participants in the experimental group who can achieve "BCl literacy" (classification accuracy of at least $80 \%$ ) for a given $\mathrm{BCl}$ type. The adoption rate does not include an element of choice, preference or selection on behalf of the subjects.

Guger et al. performed an inquiry into how many people can use an SMR-BCl (in other words are "SMR-BCl literate"). ${ }^{8}$ These authors examined the ability of subjects $(n=99)$ to imagine right- and left-hand movements to control the shift of a computer cursor on a screen in the direction of the imagined movement. Although $93 \%$ of subjects achieved a classification accuracy above $60 \%$, only $19.2 \%$ of the subjects were able to complete the task with a classification accuracy between 80-100\%. Similarly, Yao et al. revealed that only $69.7 \%$ of 43 subjects achieved a classification accuracy of at least $70 \%$ with a two-class somatosensory and motor imagery SMR-BCl. ${ }^{21}$ The group average performance in this study was $77.2 \% \pm 13.3 \% .{ }^{21}$ Although this finding by Yao et al. is not a direct comparison of $\mathrm{BCl}$ literacy rates reported by Guger et al. (2003), it nevertheless demonstrates the same preponderance of SMR-BCl users for lower adoption rates. ${ }^{8,21}$

A recent study by Chholak et al. offered greater promise for the widespread application of SMR-BCl. ${ }^{68}$ The authors proposed a more sophisticated motor imagery classification algorithm that may improve SMR-BCI performance. MEG experiments performed by $\mathrm{Ch}$ holak et al. in healthy participants confirmed the presence of two distinct types of motor imagery-related brain activity. The investigators distinguished these distinct waveforms by the patterns of activation and inhibition of different brain regions containing motor-related alpha- and beta-frequency electromagnetic signals. These authors detected two types of signals related to kinesthetic imagery and visual imagery. ${ }^{68}$ Kinesthetic imagery, in this case, is linked to the muscular sensation during the motor imagery task. Such imagery is associated with the event-related desynchronization triggered by the motor imagery task. Visual imagery is defined as the visualization of an action that leads to event-related synchronization of the electromagnetic brain activity in alpha- and beta-frequency ranges. MEG during left and right-handed MI task trials revealed a preponderance of visual imagery activity. Chholak et al. proposed the application of appropriate filtration techniques to select visual imagery as the main type of motor imagery in untrained users. ${ }^{68}$

The SMR-BCl adoption rates reported by Guger et al. (2009) and Guger et al. (2012) are significantly less than those previously reported in the $\mathrm{P} 300-\mathrm{BCl}$ and SSVEP-BCl studies. ${ }^{6,7}$ For instance, in the P300-BCl study, $76.3 \%$ of participants with a single character (SC) paradigm and $89 \%$ of participants with a row/ column (RC) paradigm achieved $\mathrm{BCl}$ literacy. Furthermore, in the SSVEP-BCI study, the authors reported an even greater adoption rate of $96.2 \% .{ }^{6}$ In conclusion, the Guger et al. series of studies determined that SMR-BCls had the lowest adoption rate while SSVEP-BCls provided the highest adoption rates among the evaluated SSVEP-BCl, P300-BCI and SMR-BCI types. ${ }^{6-8,14}$ (Figure 3 provides a summary of these findings for further evaluation and comparison.) As a result, SMR-BCl is more limited in its usability compared to other $\mathrm{BCl}$ types due to its lower adoption rates.

The adoption rates discussed above are consistent with the neurophysiology literature concerning the relative prevalence of SSVEP, P300 and SMR responses. ${ }^{12,17}$ SSVEPs are more easily elicited than P300 and SMR. ${ }^{13,22}$ SSVEP is the earliest and most automatic response. P300 and SMR are more cognitive responses, which makes them not as straightforward to elicit. Conversely, the P300 response is more difficult to elicit than SSVEP. P300 is a cognitive evoked potential that is not uniformly produced among all subjects. ${ }^{18}$ In fact, P300 is not produced at all in some individuals..$^{19}$ On the other hand, SSVEP is more uniform in its distribution amongst potential $\mathrm{BCl}$ users. ${ }^{20}$ Likewise, the experimenters discovered a lower adoption rate among $\mathrm{BCl}$ users for P300 BCls. Moreover, the subjects' capacity for abstract and imaginative thought fluctuates wildly on an individual basis. As a result, SMR-BCls had the lowest adoption rates because they require the subject's involvement in the most difficult operational task among all $\mathrm{BCl}$ types that have been studied by Guger et al. ${ }^{6-8,14}$ 


\section{BCI Adoption Rates for Different BCI Types}

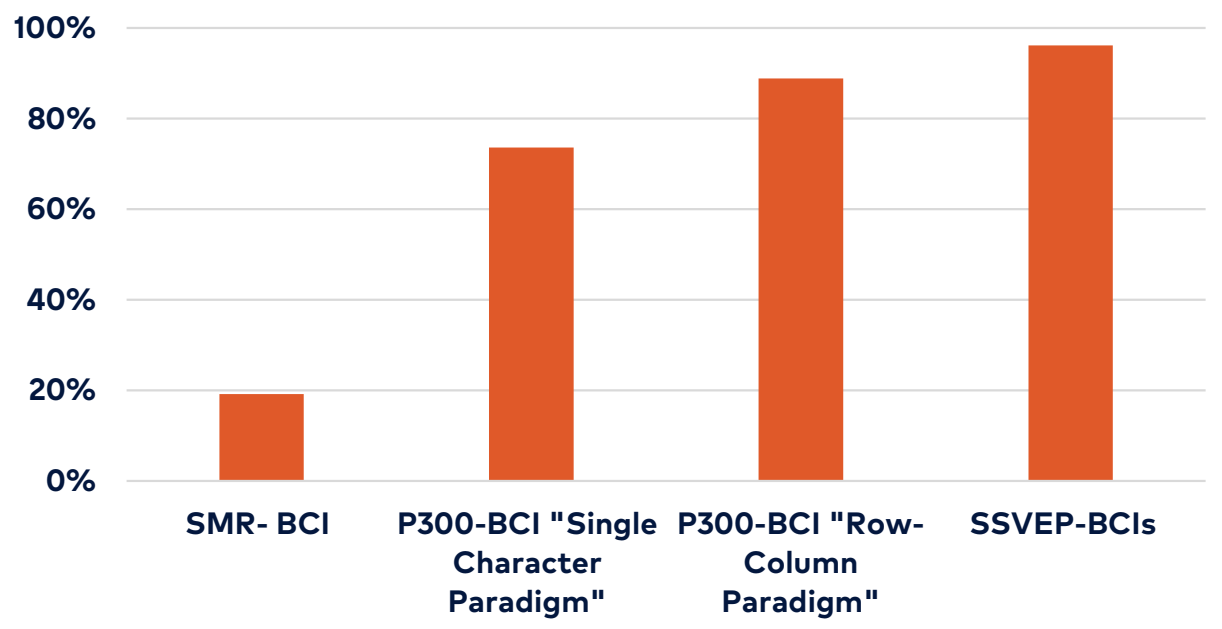

Figure 3. BCl adoption rates for different BCl types described by Guger et al. (2012); Guger et al. (2009); Guger et al. (2003); Guger et al. (2015). (-8,14 SMR-BCl was driven by simple left and righthand motor imagery tasks. $\mathrm{P} 300 \mathrm{BCl}$ and SSVEP-BCl involved spelling task. $\mathrm{BCl}$ adoption rate was defined as the proportion of $\mathrm{BCl}$ users who achieved literacy during the completion of an operational $\mathrm{BCl}$ task. ${ }^{8}$ Adoption rate was used as a measure of proficiency. It contained no elements of desire, selection, or choice. The "BCl literacy" here was defined as achieving a classification accuracy of at least $80 \%$.

\subsection{Distractors}

While in the laboratory setting, the effect of distractors is minimized. However, the real world is not a quiet place. Sidewalks are filled with the clattering of footsteps. Streets are replete with the honking of horns and screeching of tires. Distractors are a part of our living environment. They create noise that can potentially affect the performance of a $\mathrm{BCl}$ system by decreasing the signal-to-noise ratio. At the same time, distractors may affect the $\mathrm{BCl}$ user by altering their brain activity, further confounding the user's SMR-BCl performance. Serving as external factors influencing $\mathrm{BCl}$ performance, distractors are closely related to a subject's distractibility, which is an internal variable. When considering the everyday application of $\mathrm{BCl}$ technology, algorithms must be developed to account for settings beyond the laboratory. Therefore, algorithm development is crucial for the real-world application of SMR$\mathrm{BCl}$ and the assessment of SMR-BCl performance in a real-world context. ${ }^{23}$

Friedrich et al. explored the effect of auditory distractors on the performance of a cue-guided, four-class $\mathrm{BCl}$ operated by four different mental tasks: word association, mental subtraction, spatial navigation and motor imagery. ${ }^{5}$ This study demonstrated that auditory dis- tractors had no adverse effect on cue-guided, four-class hybrid P300-SMR-BCl performance. The subjects maintained their SMR-BCl performance during all of the auditory distractors. Both passive and active distraction, as well as absent distraction control conditions, were simulated. The auditory stimuli were presented in an oddball paradigm. Friedrich et al. intended passive distraction to represent background noise. To simulate passive distraction, the authors instructed subjects $(n=14)$ to ignore all tones presented in the "oddball" series. ${ }^{5}$ To simulate active distraction, the experimenters required subjects to respond with a button press to the target tone of the "oddball" paradigm. Active distraction recreated a multitasking condition in the real world. Surprisingly, passive auditory distractors optimized four-class hybrid P300-SMR-BCl performance during different mental tasks when compared to active distractors and absent distractors. This finding only offers further encouragement for the prospect of everyday $\mathrm{BCl}$ use. ${ }^{5}$

Friedrich et al. suggested that the Yerkes-Dodson law supported these findings. The current literature supports this conclusion. $., 24,25$ Researchers have successfully applied the Yerkes-Dodson law to numerous and diverse settings. ${ }^{25}$ The Yerkes-Dodson law is a psy- 
chology concept that states moderate arousal can improve performance via the modulation of motivation, but high levels of arousal can impair performance due to a reduction in the quantity of cognitive information processing. ${ }^{25}$ Likewise, passive auditory distraction improved $\mathrm{BCl}$ performance. Conversely, active auditory distraction impaired $\mathrm{BCl}$ performance since it overwhelmed, divided and diverted attentional resources from the main goal, which was initially directed towards the SMR-BCl operation.

Emami and Chau further explored the influence of distractors by conducting a study of the relationship between visual distractors and SMR$\mathrm{BCl}$ classification accuracy. ${ }^{26}$ Infrequent, small visual distractors altered mu and beta power of motor imagery-specific electrical brain activity but did not significantly alter SMR-BCl classification accuracy. Participants achieved a mean classification accuracy of $81.5 \pm 14 \%$ for non-distractor trials and $78.3 \pm 17 \%$ for distractor trials. ${ }^{26}$ These developments are promising for the everyday application of $\mathrm{BCls}$ in chaotic, real-world contexts.

\subsection{Training}

One of the stated goals for this review is to analyze methods for the optimization of SMR$\mathrm{BCl}$ performance. Life experience and anecdotal evidence can attest to the significance of practice in the mastery of a skill. For this reason, a discussion of the effect of external variables on SMR-BCl performance would be incomplete without consideration of training. For this section, we will only consider the binary presence or absence of training and its effect on SMR-BCl performance. The following sections-1.4 Visual and Auditory Feedback, 1.5 Virtual Reality and Magnetoelectric Feedback and 1.6 Proprioceptive and Haptic Feedbackwill consider a more nuanced review of training paradigms. It is anticipated that training would have a positive effect on SMR-BCl performance. Training could be an essential factor in the adoption of SMR-BCl use among healthy and disabled users. With training, SMR-BCl users who do not demonstrate immediate $\mathrm{BCl}$ literacy would not become abandoned. Instead, training would improve SMR-BCl proficiency to accepted levels of competency, thus extending the scope of SMR-BCl use beyond those who were already sufficiently skillful at the initial stages of working with SMR-BCl.

Indeed, research has affirmed the effect of training on SMR-BCl performance. ${ }^{28-31}$ Meng and $\mathrm{He}$ suggested that training sessions could lead to significant behavioral performance alteration and changes in event-related desynchronization lateralization within only a few hours. ${ }^{29}$ The results of their study revealed a significant increase in the group average SMR$\mathrm{BCl}$ classification accuracy and information transfer rate just over three sets of training sessions. Multiple training sessions may be particularly useful for SMR-BCl users who initially struggle. ${ }^{28,29}$ Pichiorri et al. showed that SMR$\mathrm{BCl}$ training led to a significant increase in the amplitude and volume of the motor potential recorded from the opponens pollicis. ${ }^{30}$ Toppi et al. examined the effects of training on electrical brain activity. ${ }^{31}$ The authors identified unique specific spectral and spatial cortical activity patterns in response to a simple motor imagery training task (e.g., the open-close motion of the hand). More complex motor imagery tasks (e.g., playing tennis) elicited moderately generalized effects on electrical brain activity. These enhanced cortical activity benefits extended long-term, further emphasizing the significant role that training could play in the widespread adoption of SMR-BCl technology. ${ }^{31}$

\subsubsection{Visual and Auditory Feedback}

Research on the effect of training on the performance of different $\mathrm{BCl}$ types has continued into the realm of SMR-BCls and sensory feedback. More evidence of the effect of SMR-BCl training came from the finding that the brain network changed its topology in response to neurofeedback, leading to enhanced SMR-BCI performance. ${ }^{30}$ Angulo-Sherman and Gutierrez further described the effect of SMR-BCl performance on electroencephalographic activity. ${ }^{32}$ Results demonstrated a high correlation between event-related coherence and SMR$\mathrm{BCl}$ performance with classical visual feedback, auditory feedback or functional electrical stimulation feedback. ${ }^{32}$ Thus, elevated motor cortical excitability, functional brain network analysis and enhanced event-related coherence served as the neurophysiological evidence for improved SMR-BCl performance with neurofeedback. 


\section{Visual Feedback}

The effect of visual feedback on SMR-BCl performance is an area of investigation. Miller et al. observed that motor imagery was associated with a level that constituted only $25 \%$ of the total magnitude of cortical activity associated with motor task execution. ${ }^{36}$ Visual feedback amplified the degree of motor cortex activation associated with mental imagery to levels comparable with, and even higher than, an actual motor movement task. Miller et al. also offered several explanations of potential mechanisms underlying the visual feedback phenomenon. ${ }^{36}$ The authors suggested that this altered pattern of cortical activation may have been a result of motor imagery's direct attempt to recruit a subset of the neuronal population. This recruitment primes those neurons immediately responsible for the transmission of motor commands to the body, facilitating more responsive $\mathrm{SMR}-\mathrm{BCl}$ performance in users. Alternatively, the authors proposed that enhanced cortical activation may have been a result of motor imagery's ability to initiate a gain in the firing rate of large motor cortical neurons.

Sollfrank et al. explored the role of enriched feedback in SMR-BCl performance." Enriched funnel feedback (EFF) may better support the initial SMR-BCl training phase than the conventional cursor bar paradigm. In addition to the left and right classification of a left- or righthand motor imagery task for the control of an onscreen cursor, the EFF paradigm provided the SMR-BCl user with visual information representing the strength of the signal for $\mathrm{BCl}$ user's control. In the EFF paradigm, a liquid cursor began at the top of the visual display in a funnel that was connected at the bottom to a test tube. The movement of the liquid cursor through the funnel to the left or right end of the test tube represented the left/right SMR-BCl classification like the cursor bar (CB) feedback. Also, this EFF paradigm provided the SMR-BCl user more visual information beyond that supplied by the CB paradigm. Specifically, the liquid cursor was initially an amorphous, diffuse collection of droplets that coalesced into a single, uniform blue sphere. This transition represented the $\mathrm{BCl}$ user's control stability."

The training effect of visual feedback has been supported by other studies. Orand et al. ob- served a significant improvement in motor imagery learning in SMR-BCl users who received abstract visual feedback. ${ }^{38}$ Conversely, users who received no visual feedback did not demonstrate a significant motor imagery learning effect. ${ }^{38}$ Ono et al. provided several forms of realistic visual feedback to $\mathrm{BCl}$ users who had previously received no visual feedback. ${ }^{37}$ These authors proposed visual feedback in three novel forms: changing bar length, hand open/grasp picture animated at the level of the SMR-BCl user's eye and the same hand picture overlaying the tested hand. ${ }^{37}$ Zich et al. assessed the effect of visual feedback in the form of real-time EEG monitoring on motor imagery activity. ${ }^{39}$ Results indicated significantly enhanced motor imagery task-specific brain activity during feedback conditions relative to no EEG monitoring feedback. ${ }^{39}$

\section{Auditory Feedback}

Auditory feedback may also improve SMR-BCl performance, either independently from visual feedback or combined with it. McCreadie et al. (2012) demonstrated that SMR-BCl users who received visual feedback performed better than those who received auditory feedback. However, this effect diminished over several training sessions. ${ }^{34}$ In contrast, SMR-BCl users presented with initial auditory feedback demonstrated consistent and sustained enhancements of average classification accuracy and average peak classification accuracy. ${ }^{35}$ The exact technology behind auditory feedback is not a significant concern. No variation in SMR-BCl performance was observed with distinct audio technologies such as mono, stereo or 3-D auditory feedback. ${ }^{35}$

Sollfrank et al. investigated the effect of auditory feedback in combination with the EFF paradigm." The researchers termed this new paradigm multimodal funnel (MF) feedback. They observed an insignificant difference in SMR-BCl performance between EFF and MF feedback. The researchers reasoned that perhaps visual feedback was too dominant for simultaneous auditory feedback to contribute to enhanced SMR-BCl performance. "The Yerkes-Dodson law supports the authors' conclusion that multimodal feedback overwhelmed SMR-BCl users. ${ }^{25}$ Expanding upon the findings presented above, Brumberg et al. described a significant improvement of SMR-BCl classi- 
fication accuracy, distance to the target and movement time to the target with multimodal feedback relative to unimodal audio or visual feedback. ${ }^{33}$ The authors concluded that optimal SMR-BCl performance may be achieved when multimodal feedback is consistent with SMR$\mathrm{BCl}$ task goals. In contrast, multimodal feedback is not effective as a generic biofeedback signal. 33

\subsubsection{Virtual Reality and Magnetoelectric Feedback Virtual Reality Feedback}

Vourvopoulos et al. investigated the use of virtual reality (VR), an emerging modality for SMR-BCl training. ${ }^{49}$ The results of a stroke rehabilitation case report detailed an increase in the intensity of Ml-related brain activity following a three-week intervention of ten $\mathrm{BCl}-\mathrm{VR}$ training sessions. In this case report, a 60-yearold male stroke patient performed a BCl-VR task in a self-paced, first-person $\mathrm{BCl}$ game. The patient applied motor imagery to a boat rowing task to collect as many flags as possible during a timed event. Ambient environmental sounds and goal sounds provided auditory feedback, and the vibrating motors inside cylindrical tubes for grasping provided haptic feedback. ${ }^{49}$ These data are promising for the future application of virtual reality feedback used with SMR-BCls. Huang et al. currently seek to perform a randomized controlled trial to further evaluate the efficacy of immersive VR in stroke rehabilitation patients and detail the underlying brain. ${ }^{43}$ Extending the potential benefits of virtual reality beyond stroke rehabilitation, Burin et al. developed a study protocol for a randomized controlled trial to evaluate the physical, cognitive and neural benefits of virtual reality training in healthy adult volunteers. ${ }^{40}$

\section{Magnetic Stimulation Feedback}

The influence of repetitive transcranial magnetic stimulation is another area of investigation for SMR-BCl research. Studies have established the ability of high-frequency, low-frequency and/or combined repetitive transcranial magnetic stimulation (rTMS) to restore superficial brain wave activity at the lesion site in patients who suffered a stroke. ${ }^{42,45,46,48}$ These findings suggest that rTMS should improve the user's SMR-BCI performance. Indeed, Shu et al. (2018a) observed an improvement in
SMR-BCl performance in stroke patients with high-frequency rTMS. ${ }^{47}$ Following 12 sessions of $10 \mathrm{~Hz}$ rTMS interventions over four consecutive weeks, the results yielded a significant enhancement relative to controls who received no $\mathrm{rTMS}$. SMR-BCl accuracy improved from $63.5 \%$ to $74.3 \%$ in $\mathrm{Ml}$ tasks and $81.9 \%$ to $91.1 \%$ in motor execution tasks. ${ }^{47}$

Johnson et al. first described the combined effect of rTMS and a virtual reality SMR-BCl in stroke rehabilitation. ${ }^{44}$ Results demonstrated significant improvements in motor activity and behavioral function. The study included two groups of participants. The treatment group consisted of participants status post-stroke who received motor rehabilitation with $V R$ and rTMS. The control group only received VR feedback. Control participants also demonstrated enhancement of motor activity and behavioral function, albeit not as significant as the patient group. ${ }^{44}$ These findings support the future use of rTMS for improved SMR-BCl performance.

\section{Electrical Stimulation Feedback}

Yi et al. (2017) sought to enhance SMR-BCI performance by incorporating electrical stimulation sensory feedback. ${ }^{50}$ Electrical stimulation induces steady-state somatosensory evoked potential (SSSEP). The authors noted that a combination of SMR-induced event-related desynchronization and SSSEP led to a significant $14 \%$ improvement in SMR-BCl classification accuracy during a hybrid task composed of motor imagery and selective attention elements. The control group involved participants who performed the motor imagery task without any associated SSSEP. The hybrid task elicited additional SSSEP beyond that seen with only electrical stimulation. Users achieved an $89 \%$ mean classification accuracy. ${ }^{50} \mathrm{~A}$ series of studies with recoveriX confirmed this high mean classification accuracy. RecoveriX is a hybrid, two-class $\mathrm{BCl}$ guided by SMR activity and electrical stimulation sensory feedback. ${ }^{15,41}$ Figure 4 provides a visual representation of this novel $\mathrm{BCl}$ system. The findings of $Y i$ et al. suggest the development of a novel hybrid SMR-SSSEP $\mathrm{BCl}$ would lead to significantly better SMR-BCI performance..$^{50}$ In summary, visual, auditory and electrical feedback can play an important role in SMR-BCl training, and, therefore, enhance SMR-BCl performance. 


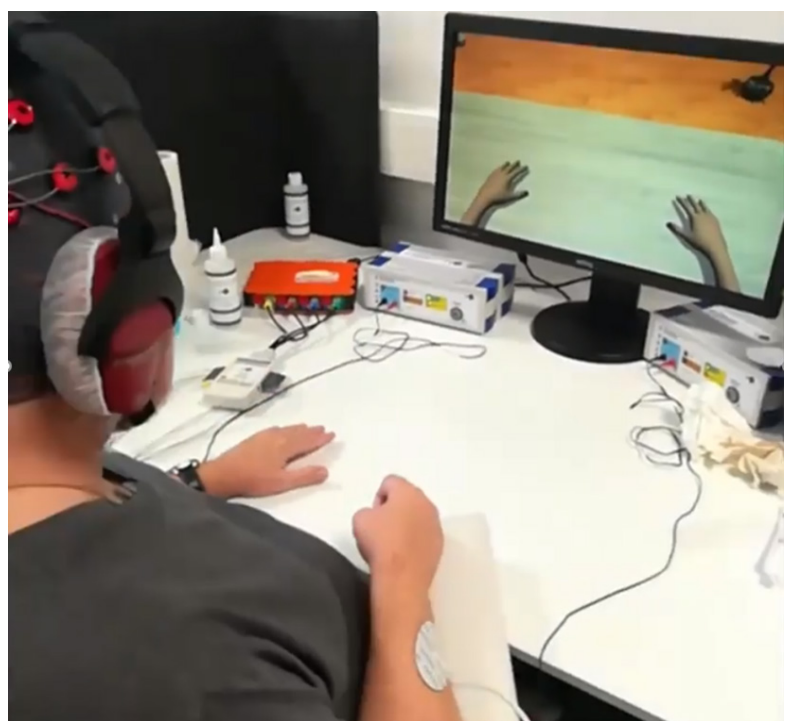

Figure 4. The SMR-BCI system (recoveriX) for upper extremity motor recovery in patients status post-stroke. It is a hybrid two-class BCI based on SMR activity and electrical stimulation sensory feedback. Motor recovery in stroke patients is an emerging application. This SMR-BCl system for rehabilitation consists of several components: Electroencephalography system (EEG); Avatar ("virtual reality"); Functional electrical stimulation (FES). While completing a motor imagery task, recoveriX provides patients with visual feedback through a virtual avatar and simultaneous tactile stimulation through electrical muscle stimulation. (Photograph courtesy of the authors.)

\subsubsection{Proprioceptive and Haptic Feedback Proprioceptive Feedback}

Nakayashiki et al. attempted to describe a neurophysiological mechanism of proprioceptive feedback. ${ }^{53}$ They noted that the strength of an event-related desynchronization associated with motor imagery varied with the change in hand positions. This was reflected either in the motor planning process or the resultant shifts of proprioception. The strength of an event-related desynchronization indicates the power of the SMR signal for $\mathrm{BCl}$ interpretation. For this reason, Nakayashiki et al. proposed that proprioceptive feedback can influence SMR-BCl performance..$^{53}$

The effect of proprioceptive feedback is not only robust but is also more significant than the effect of visual feedback on SMR-BCl performance. Darvishi et al. examined the effect of proprioceptive feedback as provided by two mechanical hand orthoses that responded to the motor imagery task of the user. ${ }^{51}$ The researchers characterized this relationship by the superior gain of task-related spectral perturbations in the alpha and beta-band. In particular, proprioceptive feedback facilitated motor imagery-related operant learning of SMR beta-band modulation. Also, enhanced
SMR-BCl performance with proprioceptive feedback occurred through the neurophysiological mechanisms of enhanced accuracy and duration of acquired brain self-modulation. These changes only appeared in the beta-frequency band. ${ }^{51}$ Vukelic and Gharabaghi observed similar findings. ${ }^{56}$ The researchers demonstrated an advanced degree of functional coupling of theta and beta-band modulation during a motor imagery task with proprioceptive feedback as compared to a motor imagery task with visual feedback. ${ }^{56}$

Ramos-Murguialday et al. demonstrated a significant positive effect of motor imagery on an $\mathrm{SMR}-\mathrm{BCl}$ performance, specifically a $\mathrm{BCl}$-driven robotic arm control. ${ }^{10}$ The researchers showed significant improvements across several performance metrics: percent of the time the robotic arm moved, maximum consecutive seconds the robotic arm moved, number of robotic arm onsets and the classical reaching target accuracy. The authors defined classical reaching target accuracy as a successful trial is defined as the cursor is in the upper half of the screen upon completion. The only measured SMR-BCl performance that did not reach a significant enhancement was the robotic arm latency time of the first movement. Ramos-Murguialday et al. also observed a substantial between-ses- 
sions group learning effect of motor imagery with and without proprioceptive feedback on several SMR-BCl metrics. ${ }^{10}$ The study published by Wang et al. supported this positive association between proprioceptive neurofeedback and SMR-BCl classification accuracy. ${ }^{57}$ These authors demonstrated significantly increased cortical activations as measured by absolute event-related desynchronization powers and real-time lateralized event-related desynchronization patterns. Also, increased mean classification accuracies and the activity of partial directed coherence-based functional connectivity networks further supported the conclusion that proprioceptive feedback led to improved SMR-BCl performance. ${ }^{57}$ Partial directed coherence is a multivariate brain connectivity estimator that represents patterns of links in the brain. Penaloza et al. further described the influence of a different neuroprosthesis on SMR activity: a human-like android robot (Geminoid $\mathrm{HI}-2) .^{54}$ Findings suggested that android feedback-based SMR-BCl training enhanced modulation of motor imagery-related EEG activity. ${ }^{54}$

\section{Haptic Feedback}

Haptic feedback is another area of investigation for SMR-BCl research. The promising results of visual and auditory feedback led investigators to evaluate the effect of additional sensory modalities on SMR-BCl performance. Shu et al. (2018b) observed a significant increase in SMR-BCl decoding accuracies in participants' status post-stroke who received tactile stimulation at the wrist relative to control participants who did not receive tactile stimulation. ${ }^{55}$ With tactile stimulation during a motor attempt task, participants' statuses post-stroke achieved $85.1 \%$ decoding accuracy. On the other hand, control participants who only performed the motor attempt task achieved a $74.5 \%$ signal decoding accuracy. ${ }^{55}$ Researchers have expanded on the work of Shu et al. (2018b), focusing on haptic stimulation. Missiroli et al. explored the role of haptic stimulation feedback's anatomic site for the SMR-BCl-based operation of a hand exoskeleton..$^{52}$ Relative to the wrist, a higher density of Meissner's and Pacinian corpuscles mechanoreceptors is found at the fingertips, which is associated with their greater sensory role. Missiroli et al. anticipated that haptic stimulation at the fingertips would improve the effect of proprioceptive feedback relative to its effect at the wrist. ${ }^{52}$ While performing hand grasping motor imagery tasks, study participants did not demonstrate a significant change in SMR$\mathrm{BCl}$ proficiency with vibration at the fingertips relative to control participants who received haptic stimulation at the wrist. ${ }^{52}$

\subsection{Carefulness of EEG Assembling and Positioning of EEG Electrodes}

Signal acquisition is an important component of the closed-loop BCl operatFion system. Brain activity for $\mathrm{BCl}$ control can be recorded with high temporal precision (millisecond resolution) by a set of sensors when they use magnetoencephalography (MEG). This process uses a set of electrode arrays placed on the scalp that employ electroencephalography (EEG). Electrode grids are also placed directly on the cortical surface when utilizing electrocorticography (ECoG). ${ }^{1}$ Figure 5 summarizes these recording modalities. For this review article, we focus our discussion on signal acquisition with EEG electrodes. The assembly, attachment and positioning of these EEG electrodes are significant considerations for ensuring signal integrity and the logistics of everyday SMR-BCl use. ${ }^{63}$

SMR-BCl users report issues concerning the bulky size of larger EEG assembly caps. ${ }^{60}$ Complications of electrode placement involve skin preparation and the use of conductive gels. ${ }^{65}$ Dry-electrode EEG systems have been developed to eliminate the need for lubri-

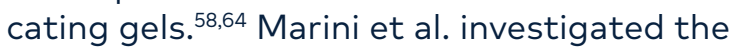
use of dry-electrode mobile EEG systems as a viable alternative to those with traditional wet-electrodes. ${ }^{61}$ Researchers concluded that the dry-electrode system performed at levels comparable to the ones with wet-electrodes. Both systems exhibited similar power spectral densities and alpha rhythm suppression during an eyes-open condition. ${ }^{61}$

Electrode placement may also play an important role in optimal $\mathrm{BCl}$ performance. In their transcranial magnetic stimulation (TMS)-guided application of EEG electrodes study, Hänselmann et al. identified a trend towards a consistent distance between the hand motor area and the site of mu-rhythm modulation for optimal EEG-recording electrode placement in SMR-BCls. ${ }^{59}$ The exact nature of this consistent 


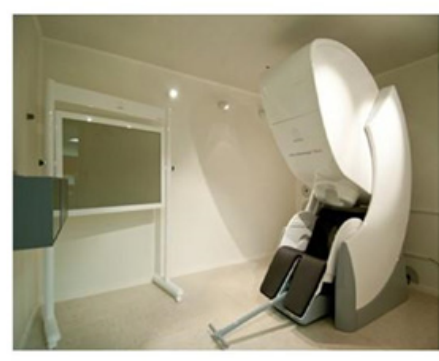

MEG

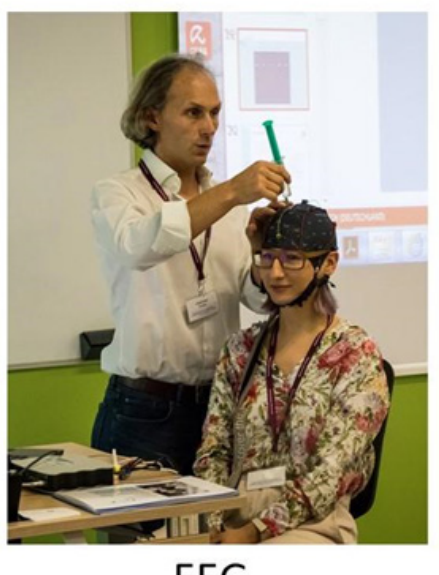

EEG

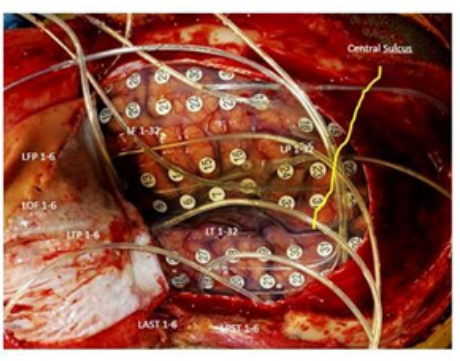

ECoG

Figure 5. Recording of magnetic (MEG) and electric (EEG, ECoG) brain activity that can be used for Brain-Computer Interface (BCl) applications. Left: Example of magnetoencephalography (MEG) at MEG Lab, AdventHealth for Children Orlando; Middle: Example of electroencephalography (EEG) at the Department of Biophysics, Vilnius University; Right: Example of electrocorticography (ECoG) at the Comprehensive Epilepsy Surgery Center, AdventHealth Orlando. (Photographs courtesy of the authors.)

distance varied on an individual basis, but it is more prevalent in the mediolateral than the anterior-posterior direction. ${ }^{59}$

Solutions have been offered to eliminate the assembly and conductive gel concerns of SMR$\mathrm{BCl}$ users such as a smart EEG cap. ${ }^{60,65}$ Lin et al. developed a spatial filtering circuit with novel dry active electrodes to enhance EEG features in a local area and to optimize EEG channel selection automatically. ${ }^{60}$ These developments led to a reduction in the number of necessary electrodes in the assembly of the smart EEG cap, mitigating the previously described size and bulk concerns. The authors combined the smaller EEG assembly size with wireless transmission to encourage portability and convenience of use. An information transfer rate of about 6.06 bits $/ \mathrm{min}$ validated the design of this smart EEG cap. ${ }^{60}$

Zhang et al. proposed an alternative portable brain-computer interface solution. ${ }^{65}$ Dry electrodes acquire the user's sensorimotor signal. This signal is transmitted to the portable $\mathrm{BCl}$. The authors developed a three-dimensional, novel, convolutional neural network using time as two-dimensions and the frequency band of the EEG signals. Their results demonstrated a significant improvement of classification performance in their proposed SMR-BCl design relative to the classification performance of current methods. These results support the use of their proposed SMR-BCl design as a viable alternative to traditional approaches. ${ }^{65}$ The conclusions of Lin et al. and Zhang et al. are encouraging for the future widespread application of mobile SMR-BCls for everyday use among both healthy and disabled users.

The findings of Raduntz and Meffert describe the limitations of current mobile electroencephalography devices. ${ }^{62}$ Among seven mobile EEG designs with wireless signal transmission, subjects demonstrated no clear preference in their visual perception of the devices' headset designs. Despite this finding, subjects were not willing to accept less comfort for a more appealing headset design. A significant change in maximal possible wearing duration further supported this conclusion. The authors detailed an exchange of enhanced signal quality and reduced artifacts with reduced convenience among mobile EEG devices. They identified a significant positive association between gel electrodes and attitude toward technology with practicability. ${ }^{62}$

\subsection{Recording-Related Artifacts}

Artifacts obfuscate the interpretation of EEG signals, thereby negatively impacting the interpretation and performance of an SMR$\mathrm{BCl} .{ }^{9}$ The ease of over the scalp EEG recording compared to invasive recording (e.g., ECoG. Refer back to Figure 5 for a representation of all recording modalities.) renders this technology more susceptible to artifacts such as environmental interference, electromyographic and 
electrooculographic activity than other recording electrode types like electrocorticography ${ }^{67}$

Winkler et al. explored the correlation between artifacts and SMR-BCls. ${ }^{66}$ The investigators developed an automatic classification algorithm to identify and remove most artifactual components identified via an independent component source analysis. The users' SMR$\mathrm{BCl}$ performance maintained consistency with pre-optimized linear classifier values when up to $60 \%$ of the EEG artifacts waveforms were removed. These data imply that Winkler et al. were successful in their pursuit of an automated solution for artifact removal and similar solutions can be used for optimizing SMR-BCl performance in a real-world context. ${ }^{66}$

Frolich et al. sought to augment SMR-BCl performance. ${ }^{27}$ In this study of artifact type, the findings suggested that only muscle artifacts negatively influenced the SMR-BCl error rate when using 119 EEG channels. However, investigators eliminated this association with an electrode array of 48 centrally located EEG channels. For the optimization of SMR-BCl performance, Frolich et al. recommended regularizing EEG assessment against muscle artifacts. ${ }^{27}$

\section{Limitations and Future Perspectives}

Many opportunities exist to expand and iterate upon the research performed by Guger and associates. A series of Guger et al. studies determined that $\mathrm{BCl}$ adoption rates were greatest with steady-state visual evoked potential (SSVEP), less with P300 and least with SMR-BCls. ${ }^{6-8,14}$ Opportunities exist to discover the adoption rates associated with other $\mathrm{BCl}$ types. Beyond $\mathrm{BCl}$ type, sample size is a concern. The subdivision of the $\mathrm{P} 300 \mathrm{BCl}$ into single character and row/column paradigms exacerbated the effect of the limited number of participants in the Guger et al. (2009) study. Increasing the sample size would have provided the experimenters with the opportunity to investigate the association between subject diversity and $\mathrm{BCl}$ adoption rates. More participants would have allowed for further inquiry into the effects of various internal factors and other external factors on the performance of different $\mathrm{BCl}$ types.
Several limitations are present in the Friedrich et al. study. ${ }^{5}$ This study demonstrated that auditory distractors had no adverse effect on cue-guided, four-class hybrid P300-SMR-BCl performance. First, the study should be repeated with a larger sample size. Next, future studies should include individuals with severe motor impairment. The inclusion of this population would allow for the results to have more direct application to contemporary clinical $\mathrm{BCl}$ users. Third, the study utilized standardized tones that were used to measure distraction instead of complex, real world noise. Standardized tones should be replaced with real-world noise to explore the impact of sound beyond the laboratory setting more accurately. Such improvements in study design could provide a greater application for $\mathrm{BCl}$ use in the real world. The effect of auditory distractors on other types of $\mathrm{BCls}$ should also be explored further to compare the effect among them. Infrequent, small visual distractors altered $\mathrm{mu}$ and beta power of motor imagery-specific patterns but did not significantly alter SMR-BCl classification accuracy. ${ }^{26}$ More research is needed to confirm this insignificant effect of visual distractors on SMR-BCl performance. Friedrich et al. also noted that discrete feedback was provided at the end of each distraction trial, which can become a separate area of investigation to explore the effects of feedback on $\mathrm{BCl}$ performance. ${ }^{5}$

Sollfrank et al. identified several study areas for future growth." The researchers cautioned against wholly attributing the improved initial SMR-BCl performance to the cursor bar of the enriched funnel feedback and multimodal funnel feedback paradigms." An alternative explanation could be due to the lack of online data inclusion. Online $\mathrm{BCl}$ calibration involves EEG waveforms obtained during a session for the development of a classifier to identify future EEG waveforms. On the other hand, offline $\mathrm{BCl}$ calibration involves the collection of EEG waveforms before a session. Users theoretically deduce the identity of these waveforms for the development of a classifier. ${ }^{69}$ The inclusion of online data in the reported offline findings may lead to a different association of these feedback methods with SMR-BCl performance. Sollfrank et al. clarified that the inter-session, non-stationarity of brain patterns affected all SMR-BCl classification accuracy results, but 
the uncertainty metric of the funnel may have been more susceptible to the effect of inter-session non-stationarity." This theory would appropriately explain the decline of SMR-BCl classification accuracy values across all funnel paradigm sessions. That classification accura$c y$, with respect to $C B$, did not dwindle in this manner.

Sollfrank et al. also warned that the SMR-BCl classification accuracy results of the funnel feedback groups might not have been accurate." For SMR-BCl end-users with minimal input signal integrity, the 15 second task duration may have been too long. A higher proportion of users with funnel feedback paradigms timed out than those with the conventional CB paradigm. In the event of a time-out, researchers scored the session a miss even if the cursor was advancing in the correct direction. For this reason, the funnel feedback SMR-BCl classification accuracy values may have been skewed too low. Sollfrank et al. only used healthy subjects in this study." Future research into this area should incorporate disabled $\mathrm{BCl}$ end-users to produce results that are more relevant for modern $\mathrm{BCl}$ users.

Several opportunities exist to expand upon the findings of Ramos-Murguialday et al..$^{10}$ This study demonstrated a significant positive effect of motor imagery on an SMR-BCI performance, specifically a $\mathrm{BCl}$-driven robotic arm control. First, this study involved only healthy volunteers. Disabled users should be incorporated into the study population. Currently, a large proportion of SMR-BCl users are patients with neurodegenerative conditions or are muscular system-compromised. A study on users with disabilities would produce results with more relevance for these users. This current study only contained 23 subjects. More subjects are needed to yield findings with a greater level of significance..$^{0}$ In particular, SMR-BCls are noted for a high level of inter-subject variability. ${ }^{70,71}$ For this reason, the production of results with a strong correlation strength is of concern. An exploration of the effect of mental imagery training on the SMR-BCl performance of a different prosthesis beyond a robotic arm used in a study by Ramos Murguialday et al. might also be beneficial.

\section{Next Steps}

The domain of SMR-BCl performance optimization involves the SMR-BCl itself. While not all variables have demonstrated a positive effect, external variables have the potential to improve SMR-BCl performance metrics such as classification accuracy, information transfer rate or task duration. Gaps of knowledge remain that may or may not affect the real-world application of SMR-BCl. The sample size of a study is one of the most crucial aspects of producing significance. Below are provided some suggestions for future investigations of external variables that may affect SMR-BCl performance.

\subsection{Virtual Reality Feedback}

Current studies have evaluated the effectiveness of SMR-BCl training with virtual reality feedback for motor recovery of participants post-stroke. ${ }^{43,49}$ We propose that the next step expands on this research to explore the association of virtual reality feedback with SMR-BCl performance. Virtual reality is an emerging technology with many new medical applications. ${ }^{72-75}$ The positive effect of multimodal feedback on SMR-BCI performance relative to unimodal auditory or visual feedback in Brumberg et al. is encouraging for virtual reality feedback applications. ${ }^{33}$ With its integration of auditory, visual, kinesthetic and vibrotactile feedback, virtual reality holds promise to offer more extensive sensory feedback than previous feedback modalities, thereby further optimizing SMR-BCl performance.

\subsection{Drones}

Current studies describe the effect of SMR$\mathrm{BCl}$ performance with neuroprostheses such as a robotic arm, hand or lower body exoskeletons or full-body android. 10,52,54 While these neuroprostheses have helped the advancement of motor rehabilitation-the classic goal of SMR$\mathrm{BCl}$ research-drones as neuroprostheses hold more potential. We propose future research to describe the effect of drones on SMR-BCl performance. Drones may potentially offer not just motor rehabilitation but motor enhancement in the form of flight-a motor ability that surpasses the human body.

\subsection{Repetitive Transcranial Magnetic Stimulation (rTMS)}

More research is needed to describe further 
the effects of low-frequency and combined rTMS on SMR-BCI performance as well as the electroencephalographic activity at neural sites beyond the lesion and in healthy users. More subjects are needed to achieve these additional research goals.

\subsection{EEG Placement and Positioning}

As it was described in section 1.5 above, Hänselmann et al. identified a nondistinct trend towards a consistent distance between the hand motor area and the area of mu-rhythm modulation. ${ }^{59}$ More research is needed to elucidate the directionality of this relationship, its uniformity amongst users and its prevalence for SMR-BCl optimization.

\section{Conclusion and Future \\ Perspectives}

The goals of this review paper were (1) to integrate existing knowledge about the factors affecting SMR-BCl performance by critically examining the effects of external variables on SMR-BCl described in previously published studies, as well as (2) to discuss limitations and propose further directions for $\mathrm{Ml}-\mathrm{BCl}$ research along with other possible factors that may affect the SMR-BCls performance when presented within a real-world context.

Per these goals, we may share several conclusions about the effect of external variables on $\mathrm{SMR}-\mathrm{BCl}$ performance. $\mathrm{BCl}$ type is a significant factor when considering $\mathrm{BCl}$ performance. Patients have demonstrated the greatest adoption rate with SMR-BCls, then $\mathrm{P} 300 \mathrm{BCls}$ and, lastly, with SSVEP-BCls. These adoption rates follow the prevalence and elicitability trends of the associated waveforms. Passive auditory distraction is associated with an increase in SMR-BCl performance. At the same time, visual distractors seem not to have any significant effect on SMR-BCl performance. These findings are promising for the ecological application of SMR-BCls in a real-world context. Furthermore, auditory, visual, electrical, proprioceptive and haptic feedback individually optimize SMR-BCl performance. Repetitive transcranial magnetic stimulation also shares a similar relationship with SMR-BCl performance. The influence of multimodal feedback on SMR-BCl performance is not as clear as the effect of unimodal feedback. Multimodal feedback enhances SMR-BCI performance only when the feedback is consistent with the operational task. Current literature for the effect of virtual reality feedback on SMR-BCl performance is limited. When the effect of artifacts on $\mathrm{SMR}-\mathrm{BCl}$ is considered, the muscle artifact is negatively associated with SMR-BCl performance. In order to maintain signal integrity and mitigate the effect of muscle artifact, EEG electrodes should be arranged centrally around the cranium. Optimization of these external variables along with internal variables may help achieve the intended application of widespread everyday SMR-BCl use among healthy and disabled users. ${ }^{76}$

The current literature for the effects of external variables on SMR-BCl performance shares a significant limitation. Due to its limited availability, SMR-BCl research often includes small sample sizes. Larger sample sizes are needed to yield findings with more statistical power and evaluate the common goal of SMR-BCl performance in healthy users. We propose future perspectives of SMR-BCl research in the areas of virtual reality feedback, drones for motor enhancement, alternative modes of repetitive transcranial magnetic stimulation and optimal EEG placement and positioning for the improvement of SMR-BCl performance. SMR$\mathrm{BCl}$ research remains an exciting area of great promise for its future widespread application among both disabled and healthy users. Based on the data that we have reviewed, there are more internal rather than external variables affecting $\mathrm{BCl}$ performance. Therefore, we emphasize the need for evaluating these variables and optimizing them. We discuss the effect of internal variables on SMR-BCl performance in a separate review article. ${ }^{76}$

\section{Abbreviations}

$\mathrm{BCls}$ - brain-computer interfaces; CB - cursor bar, CRR - correct response rate; EEG - electroencephalography, EFF - enriched funnel feedback, EMG - electromyography, ERDs - event-related desynchronizations, ERPs - event-related potentials; ITR - information transfer rate; MF - multimodal funnel, MI - motor imagery; RC-row/column; rTMS - repetitive transcranial magnetic stimulation; SC- single character, SMR - sensorimotor rhythm, SSSEP- steady-state somatosensory evoked potential, SSVEPs - steady-state visually evoked potentials; VEP - visual evoked potential 


\section{Conflicts of Interest}

Dr. Christoph Guger is the CEO and owner of g.tec, a company that sells neurotechnology on the international market.

Drs. Horowitz and Korostenskaja declare they have no conflicts of interest.

Dr. Horowitz is an employee of University of Central Florida/HCA Healthcare GME Consortium, an organization affiliated with the journal's publisher.

This research was supported (in whole or in part) by HCA Healthcare and/or an HCA Healthcare affiliated entity. The views expressed in this publication represent those of the author(s) and do not necessarily represent the official views of HCA Healthcare or any of its affiliated entities.

\section{Author Affiliations}

1. Functional Brain Mapping and Brain Computer Interface Lab, Neuroscience Institute, AdventHealth Orlando, Orlando, FL, USA

2. g.tec Medical Engineering GmbH, Graz, Austria

3. MEG Lab, AdventHealth for Children, Orlando, FL, USA

4. Department of Psychology, College of Arts and Sciences, University of North Florida, Jacksonville, FL, USA

5. Comprehensive Epilepsy Center, AdventHealth Orlando, Orlando, FL, USA

6. University of Central Florida/HCA Healthcare GME Consortium, Gainesville, Florida

\section{References}

1. Korostenskaja M, Kapeller C, Chen PC, et al. Estimation of Intracranial P300 Speller Sites with Magnetoencephalography (MEG)-Perspectives for Non-invasive Navigation of Subdural Grid Implantation. In: Guger C, Allison B, Ushiba J, eds. Brain-Computer Interface Research: A Stateof-the-Art Summary 5. Springer International; 2017:111-121. http://dx.doi.org/10.1007/978-3-31957132-4 9

2. Bamdad M, Zarshenas H, Auais MA. Application of $\mathrm{BCl}$ systems in neurorehabilitation: a scoping review. Disabil Rehabil Assist Technol. 2015;10(5):355-364. https://doi.org/10.3109/17483 $\underline{107.2014 .961569}$

3. Monge-Pereira E, Ibañez-Pereda J, Alguacil-Diego IM, et al. Use of electroencephalography brain-computer interface systems as a rehabil- itative approach for upper limb function after a stroke: a systematic review. PM R. 2017;9(9):918932. https://doi.org/10.1016/j.pmrj.2017.04.016

4. Bowsher K, Civillico EF, Coburn J, et al. Brain-computer interface devices for patients with paralysis and amputation: a meeting report. J Neural Eng. 2016;13(2):023001. https://doi. org/10.1088/1741-2560/13/2/023001

5. Friedrich EV, Scherer R, Sonnleitner K, Neuper C. Impact of auditory distraction on user performance in a brain-computer interface driven by different mental tasks. Clin Neurophysiol. 2011;122(10):2003-2009. https://doi.org/10.1016/i. clinph.2011.03.019

6. Guger C, Allison BZ, Großwindhager B, et al. How many people could use an SSVEP BCl?. Front Neurosci. 2012;6:169. https://doi. org/10.3389/fnins.2012.00169

7. Guger C, Daban S, Sellers E, et al. How many people are able to control a P300-based brain-computer interface (BCI)?. Neurosci Lett. 2009;462(1):94-98. https://doi.org/10.1016/j. neulet.2009.06.045

8. Guger C, Edlinger G, Harkam W, Niedermayer I, Pfurtscheller G. How many people are able to operate an EEG-based brain-computer interface (BCl)?. IEEE Trans Neural Syst Rehabil Eng. 2003;11(2):145-147. https://doi.org/10.1109/tnsre.2003.814481

9. Nijboer F, Birbaumer N, Kübler A. The influence of psychological state and motivation on brain-computer interface performance in patients with amyotrophic lateral sclerosis - a longitudinal study. Front Neurosci. 2010;4:55. https://doi.org/10.3389/fnins.2010.00055

10. Ramos-Murguialday A, Schürholz M, Caggiano V, et al. Proprioceptive feedback and brain computer interface $(\mathrm{BCl})$ based neuroprostheses. PLoS One. 2012;7(10):e47048. https://doi. org/10.1371/journal.pone.0047048

11. Sollfrank T, Ramsay A, Perdikis S, et al. The effect of multimodal and enriched feedback on SMR-BCl performance. Clin Neurophysiol. 2016;127(1):490-498. https://doi.org/10.1016/j. clinph.2015.06.004

12. Brunner C, Allison BZ, Altstätter C, Neuper C. A comparison of three brain-computer interfaces based on event-related desynchronization, steady state visual evoked potentials, or a hybrid approach using both signals. J Neural Eng. 2011;8(2):025010. https://doi.org/10.1088/17412560/8/2/025010

13. Ding J, Sperling G, Srinivasan R. Attentional modulation of SSVEP power depends on the network tagged by the flicker frequency. Cereb Cortex. 2006;16(7):1016-1029. https://doi. org/10.1093/cercor/bhj044

14. Guger C, Kapeller C, Ortner R, Kamada K. Motor Imagery with Brain-Computer Interface Neurotechnology. In: Garcia BM, ed. Motor Imagery: 
Emerging Practices, Role in Physical Therapy and Clinical Implications. Nova Science; 2015:61-79.

15. Guger C, Ramoser H, Pfurtscheller G. Real-time EEG analysis with subject-specific spatial patterns for a brain-computer interface $(\mathrm{BCl})$. IEEE Trans Rehabil Eng. 2000;8(4):447-456. https:// doi.org/10.1109/86.895947

16. Kapeller C, Hintermuller C, Abu-Alqumsan M, Pruckl R, Peer A, Guger C. A BCl using VEP for continuous control of a mobile robot. Annu Int Conf IEEE Eng Med Biol Soc. 2013;2013:5254-5257. https://doi.org/10.1109/embc.2013.6610734

17. Kuś R, Duszyk A, Milanowski P, et al. On the quantification of SSVEP frequency responses in human EEG in realistic $\mathrm{BCl}$ conditions. PLoS One. 2013;8(10):e77536. https://doi.org/10.1371/ journal.pone.0077536

18. Malone SM, Vaidyanathan U, Basu S, Miller MB, McGue M, lacono WG. Heritability and molecular-genetic basis of the P3 event-related brain potential: a genome-wide association study. Psychophysiology. 2014;51(12):1246-1258. https:// doi.org/10.1111/psyp.12345

19. Musiek FE, Baran JA, Pinheiro ML. P300 results in patients with lesions of the auditory areas of the cerebrum. J Am Acad Audiol. 1992;3(1):5-15.

20. Srinivasan R, Bibi FA, Nunez PL. Steady-state visual evoked potentials: distributed local sources and wave-like dynamics are sensitive to flicker frequency. Brain Topogr. 2006;18(3):167-187. https://doi.org/10.1007/s10548-006-0267-4

21. Yao L, Sheng X, Mrachacz-Kersting N, Zhu X, Farina D, Jiang N. Performance of brain-computer interfacing based on tactile selective sensation and motor imagery. IEEE Trans Neural Syst Rehabil Eng. 2018;26(1):60-68. https://doi. org/10.1109/tnsre.2017.2769686

22. Zhu D, Bieger J, Garcia Molina G, Aarts RM. A survey of stimulation methods used in SSVEP-based BCls. Comput Intell Neurosci. 2010;2010:702357. https://doi. org/10.1155/2010/702357

23. Brandl S, Frølich L, Höhne J, Müller KR, Samek W. Brain-computer interfacing under distraction: an evaluation study. J Neural Eng. 2016;13(5):056012. https://doi.org/10.1088/17412560/13/5/056012

24. Calabrese EJ. Stress biology and hormesis: the Yerkes-Dodson law in psychology--a special case of the hormesis dose response. Crit Rev Toxicol. 2008;38(5):453-462. https://doi. org/10.1080/10408440802004007

25. Chaby LE, Sheriff MJ, Hirrlinger AM, Braithwaite VA. Can we understand how developmental stress enhances performance under future threat with the Yerkes-Dodson law?. Commun Integr Biol. 2015;8(3):e1029689. https://doi.org/10 $.1080 / 19420889.2015 .1029689$

26. Emami Z, Chau T. Investigating the effects of visual distractors on the performance of a motor imagery brain-computer interface. Clin Neurophysiol. 2018;129(6):1268-1275. https://doi. org/10.1016/i.clinph.2018.03.015

27. Frolich L, Winkler I, Muller KR, Samek W. Investigating effects of different artefact types on motor imagery $\mathrm{BCl}$. Annu Int Conf IEEE Eng Med Biol Soc. 2015;2015:1942-1945. https://doi. org/10.1109/embc.2015.7318764

28. Kaiser V, Bavernfeind G, Kreilinger A, et al. Cortical effects of user training in a motor imagery based brain-computer interface measured by fNIRS and EEG. Neuroimage. 2014;85 Pt 1:432-444. https://doi.org/10.1016/i.neuroimage.2013.04.097

29. Meng J, He B. Exploring training effect in 42 human subjects using a non-invasive sensorimotor rhythm based online $\mathrm{BCl}$. Front Hum Neurosci. 2019;13:128. https://doi.org/10.3389/ fnhum.2019.00128

30. Pichiorri F, De Vico Fallani F, Cincotti F, et al. Sensorimotor rhythm-based brain-computer interface training: the impact on motor cortical responsiveness. J Neural Eng. 2011;8(2):025020. https://doi.org/10.1088/1741-2560/8/2/025020

31. Toppi J, Risetti M, Quitadamo LR, et al. Investigating the effects of a sensorimotor rhythm-based $\mathrm{BCl}$ training on the cortical activity elicited by mental imagery. J Neural Eng. 2014;11(3):035010. https://doi.org/10.1088/17412560/11/3/035010

32. Angulo-Sherman IN, Gutiérrez D. A link between the increase in electroencephalographic coherence and performance improvement in operating a brain-computer interface. Comput Intell Neurosci. 2015;2015:824175. https://doi. org/10.1155/2015/824175

33. Brumberg JS, Pitt KM, Burnison JD. A noninvasive brain-computer interface for real-time speech synthesis: the importance of multimodal feedback. IEEE Trans Neural Syst Rehabil Eng. 2018;26(4):874-881. https://doi.org/10.1109/tnsre.2018.2808425

34. McCreadie KA, Coyle DH, Prasad G. Learning to modulate sensorimotor rhythms with stereo auditory feedback for a brain-computer interface. Annu Int Conf IEEE Eng Med Biol Soc. 2012;2012:6711-6714. https://doi.org/10.1109/ embc.2012.6347534

35. McCreadie KA, Coyle DH, Prasad G. Is sensorimotor $\mathrm{BCl}$ performance influenced differently by mono, stereo, or 3-D auditory feedback?. IEEE Trans Neural Syst Rehabil Eng. 2014;22(3):431440. https://doi.org/10.1109/tnsre.2014.2312270

36. Miller KJ, Schalk G, Fetz EE, den Nijs M, Ojemann JG, Rao RP. Cortical activity during motor execution, motor imagery, and imagery-based online feedback. Proc Natl Acad Sci U S A. 2010;107(9):4430-4435. https://doi. org/10.1073/pnas.0913697107 
37. Ono T, Kimura A, Ushiba J. Daily training with realistic visual feedback improves reproducibility of event-related desynchronisation following hand motor imagery. Clin Neurophysiol. 2013;124(9):1779-1786. https://doi.org/10.1016/j. clinph.2013.03.006

38. Orand A, Ushiba J, Tomita Y, Honda S. The comparison of motor learning performance with and without feedback. Somatosens Mot Res. 2012;29(3):103-110. https://doi.org/10.3109/08990 $\underline{220.2012 .687419}$

39. Zich C, Debener S, Kranczioch C, Bleichner MG, Gutberlet I, De Vos M. Real-time EEG feedback during simultaneous EEG-fMRI identifies the cortical signature of motor imagery. Neuroimage. 2015;114:438-447. https://doi.org/10.1016/i. neuroimage.2015.04.020

40. Burin D, Yamaya N, Ogitsu R, Kawashima R. Virtual training leads to real acute physical, cognitive, and neural benefits on healthy adults: study protocol for a randomized controlled trial. Trials. 2019;20(1):559. https://doi.org/10.1186/s13063019-3591-1

41. Cho W, Sabathiel N, Ortner R, et al. Paired associative stimulation using brain-computer interfaces for stroke rehabilitation: a pilot study. Eur J Trans/ Myol. 2016;26(3):6132. https://doi. org/10.4081/ejtm.2016.6132

42. de Vries PM, de Jong BM, Bohning DE, Walker JA, George MS, Leenders KL. Changes in cerebral activations during movement execution and imagery after parietal cortex TMS interleaved with 3T MRI. Brain Res. 2009;1285:58-68. https:// doi.org/10.1016/j.brainres.2009.06.006

43. Huang $Q, W u W$, Chen $X$, et al. Evaluating the effect and mechanism of upper limb motor function recovery induced by immersive virtual-reality-based rehabilitation for subacute stroke subjects: study protocol for a randomized controlled trial. Trials. 2019;20(1):104. https://doi. org/10.1186/s13063-019-3177-y

44. Johnson NN, Carey J, Edelman BJ, et al. Combined rTMS and virtual reality brain-computer interface training for motor recovery after stroke. J Neural Eng. 2018;15(1):016009. https:// doi.org/10.1088/1741-2552/aa8ce3

45. Long $\mathrm{H}$, Wang $\mathrm{H}$, Zhao $\mathrm{C}$, et al. Effects of combining high- and low-frequency repetitive transcranial magnetic stimulation on upper limb hemiparesis in the early phase of stroke. Restor Neurol Neurosci. 2018;36(1):21-30. https://doi. org/10.3233/rnn-170733

46. Pan W, Wang P, Song X, Sun X, Xie Q. The effects of combined low frequency repetitive transcranial magnetic stimulation and motor imagery on upper extremity motor recovery following stroke. Front Neurol. 2019;10:96. https:// doi.org/10.3389/fneur.2019.00096

47. Shu X, Chen S, Chai G, Sheng X, Jia J, Zhu X. Neural modulation by repetitive transcranial magnetic stimulation (rTMS) for $\mathrm{BCl}$ enhancement in stroke patients. Annu Int Conf IEEE Eng Med Biol Soc. 2018;2018:2272-2275. https://doi. org/10.1109/embc.2018.8512860

48. Töpper R, Foltys H, Mottaghy FM, Boroojerdi B. Repetitive transcranial magnetic stimulation of the parietal cortex influences motor imagery. Electroencephalogr Clin Neurophysiol Suppl. 1999;51:145-150.

49. Vourvopoulos A, Jorge C, Abreu R, Figueiredo P, Fernandes JC, Bermúdez I Badia S. Efficacy and brain imaging correlates of an immersive motor imagery $\mathrm{BCl}$-driven VR system for upper limb motor rehabilitation: a clinical case report. Front Hum Neurosci. 2019;13:244. https://doi. org/10.3389/fnhum.2019.00244

50. Yi W, Qiu S, Wang K, et al. Enhancing performance of a motor imagery based brain-computer interface by incorporating electrical stimulation-induced SSSEP. J Neural Eng. 2017;14(2):026002. https://doi.org/10.1088/17412552/aa5559

51. Darvishi S, Gharabaghi A, Boulay CB, Ridding MC, Abbott D, Baumert M. Proprioceptive feedback facilitates motor imagery-related operant learning of sensorimotor $\beta$-band modulation. Front Neurosci. 2017;11:60. https://doi. org/10.3389/fnins.2017.00060

52. Missiroli F, Barsotti M, Leonardis D, Gabardi M, Rosati G, Frisoli A. Haptic stimulation for improving training of a motor imagery $\mathrm{BCl}$ developed for a hand-exoskeleton in rehabilitation. IEEE Int Conf Rehabil Robot. 2019;2019:1127-1132. https://doi.org/10.1109/icorr.2019.8779370

53. Nakayashiki K, Saeki M, Takata Y, Hayashi Y, Kondo T. Modulation of event-related desynchronization during kinematic and kinetic hand movements. J Neuroeng Rehabil. 2014;11:90. https://doi.org/10.1186/1743-0003-11-90

54. Penaloza Cl, Alimardani M, Nishio S. Android feedback-based training modulates sensorimotor rhythms during motor imagery. IEEE Trans Neural Syst Rehabil Eng. 2018;26(3):666-674. https://doi.org/10.1109/tnsre.2018.2792481

55. Shu X, Chen S, Meng J, et al. Tactile stimulation improves sensorimotor rhythm-based $\mathrm{BCl}$ performance in stroke patients. IEEE Trans Biomed Eng. 2018;10.1109/TBME.2018.2882075. https:// doi.org/10.1109/tbme.2018.2882075

56. Vukelić M, Gharabaghi A. Oscillatory entrainment of the motor cortical network during motor imagery is modulated by the feedback modality. Neuroimage. 2015;111:1-11. https://doi. org/10.1016/j.neuroimage.2015.01.058

57. Wang Z, Zhou Y, Chen L, et al. A BCl based visual-haptic neurofeedback training improves cortical activations and classification performance during motor imagery. J Neural Eng. 2019;16(6):066012. https://doi.org/10.1088/17412552/ab377d 
58. Baek HJ, Chang MH, Heo J, Park KS. Enhancing the usability of brain-computer interface systems. Comput Intell Neurosci. 2019;2019:5427154. https://doi.org/10.1155/2019/5427154

59. Hänselmann S, Schneiders M, Weidner N, Rupp R. Transcranial magnetic stimulation for individval identification of the best electrode position for a motor imagery-based brain-computer interface. J Neuroeng Rehabil. 2015;12:71. https:// doi.org/10.1186/s12984-015-0063-z

60. Lin BS, Huang YK, Lin BS. Design of smart EEG cap. Comput Methods Programs Biomed. 2019;178:41-46. https://doi.org/10.1016/j. cmpb.2019.06.009

61. Marini F, Lee C, Wagner J, Makeig S, Gola M. A comparative evaluation of signal quality between a research-grade and a wireless dry-electrode mobile EEG system. J Neural Eng. 2019;16(5):054001. https://doi.org/10.1088/17412552/ab21f2

62. Radüntz T, Meffert B. User experience of 7 mobile electroencephalography devices: comparative study. JMIR Mhealth Uhealth. 2019;7(9):e14474. https://doi.org/10.2196/14474

63. Sannelli C, Dickhaus T, Halder S, Hammer EM, Müller KR, Blankertz B. On optimal channel configurations for SMR-based brain-computer interfaces. Brain Topogr. 2010;23(2):186-193. https://doi.org/10.1007/s10548-010-0135-0

64. Spüler M. A high-speed brain-computer interface $(\mathrm{BCl})$ using dry EEG electrodes. PLoS One. 2017;12(2):e0172400. https://doi.org/10.1371/journal.pone.0172400

65. Zhang Y, Zhang X, Sun H, Fan Z, Zhong X. Portable brain-computer interface based on novel convolutional neural network. Comput Biol Med. 2019;107:248-256. https://doi.org/10.1016/j.compbiomed.2019.02.023

66. Winkler I, Haufe S, Tangermann M. Automatic classification of artifactual ICA-components for artifact removal in EEG signals. Behav Brain Funct. 2011;7:30. https://doi.org/10.1186/17449081-7-30

67. Yuan $\mathrm{H}, \mathrm{He} \mathrm{B}$. Brain-computer interfaces using sensorimotor rhythms: current state and future perspectives. IEEE Trans Biomed Eng. 2014;61(5):1425-1435. https://doi.org/10.1109/ tbme.2014.2312397

68. Chholak P, Niso G, Maksimenko VA, et al. Visual and kinesthetic modes affect motor imagery classification in untrained subjects. Sci Rep. 2019;9(1):9838. https://doi.org/10.1038/s41598019-46310-9

69. Wu D. Online and offline domain adaptation for reducing $\mathrm{BCl}$ calibration effort. IEEE Trans Hum Mach Syst. 47(4): 550-563. https://doi. org/10.1109/THMS.2016.2608931

70. Jeunet C, N'Kaoua B, Lotte F. Advances in user-training for mental-imagery-based $\mathrm{BCl}$ control: Psychological and cognitive fac- tors and their neural correlates. Prog Brain Res. 2016;228:3-35. https://doi.org/10.1016/ bs.pbr.2016.04.002

71. Scherer R, Faller J, Friedrich EV, et al. Individually adapted imagery improves brain-computer interface performance in end-users with disability. PLoS One. 2015;10(5):e0123727. https://doi. org/10.1371/journal.pone.0123727

72. Ashmore J, Di Pietro J, Williams K, et al. A free virtual reality experience to prepare pediatric patients for magnetic resonance imaging: cross-sectional questionnaire study. JMIR Pediatr Parent. 2019;2(1):e11684. https://doi. org/10.2196/11684

73. Bakker A, Janssen L, Noordam C. Home to hospital live streaming with virtual reality goggles: a qualitative study exploring the experiences of hospitalized children. JMIR Pediatr Parent. 2018;1(2):e10. https://doi.org/10.2196/pediatrics.9576

74. Park E, Yun BJ, Min YS, et al. Effects of a mixed reality-based cognitive training system compared to a conventional computer-assisted cognitive training system on mild cognitive impairment: a pilot study. Cogn Behav Neurol. 2019;32(3):172-178. https://doi.org/10.1097/ wnn.0000000000000197

75. Zawy Alsofy S, Stroop R, Fusek I, et al. Early autologous cranioplasty: complications and identification of risk factors using virtual reality visualisation technique. $\mathrm{Br} J$ Neurosurg. 2019;33(6):664-670. https://doi.org/10.1080/0268 8697.2019.1661962

76. Horowitz A, Guger C, Korostenskaja M. What internal variables affect sensorimotor rhythm brain-computer interface (SMR-BCl) performance?. HCA Healthcare Journal of Medicine. 2021;2(3):163-179. https://doi.org/10.36518/2689$\underline{0216.1196}$ 